See discussions, stats, and author profiles for this publication at: https://www.researchgate.net/publication/339797583

\title{
Challenges of Vehicle-to-Everything Communication. Interviews among Industry Experts
}

Chapter $\cdot$ March 2020

Dol: 10.30844/wi_2020_r12-renner

\section{CITATIONS}

5 authors, including:

Maximilian Renner

Karlsruhe Institute of Technology

2 PUBLICATIONS OCITATIONS

SEE PROFILE

Sebastian Lins

Karlsruhe Institute of Technology

65 PUBLICATIONS 405 CITATIONS

SEE PROFILE
READS

26

Johanna von Hammerstein

Karlsruhe Institute of Technology

2 PUBLICATIONS OCITATIONS

SEE PROFILE

Some of the authors of this publication are also working on these related projects:

Multi-Disciplinary Identification of Lineage-Specific Signaling Dependencies in Cancer (MILES) View project

Continuous Certification of Cloud Services View project 


\title{
Challenges of Vehicle-to-Everything Communication. Interviews among Industry Experts
}

\author{
Maximilian Renner ${ }^{1}$, Niels Münzenberger ${ }^{1}$, Johanna von Hammerstein ${ }^{1}$, Sebastian \\ Lins $^{1}$ and Ali Sunyaev ${ }^{1}$ \\ ${ }^{1}$ Karlsruhe Institute of Technology, Institute of Applied Informatics and Formal Description \\ Methods (AIFB), Karlsruhe, Germany \\ \{maximilian.renner, niels.muenzenberger, johanna.hammerstein\}@stud \\ ent.kit.edu, \{sebastian.lins, sunyaev\}@kit.edu
}

\begin{abstract}
By 2025 every new car sold will be connected to the Internet, leading to a disruptive change of road transportation, but also resulting in diverse challenges regarding Vehicle-to-Everything Communication (V2X). Prior research has mostly focused on the technological challenges of V2X and neglects to consider environmental and organizational challenges resulting from a highly interconnected and interdependent market. We conducted 19 semi-structured interviews with experts from multiple market players to achieve a more holistic overview of what challenges implementation and diffusion of V2X are facing. Our findings reveal that $\mathrm{V} 2 \mathrm{X}$ is perceived as a critical information infrastructure coming with several challenges that must be tackled, such as security and privacy issues, lacking trust and acceptance, and entangled and unclear responsibilities. By synthesizing major challenges, we provide recommendations for future research avenues to overcome existing challenges and enable widespread diffusion of V2X to unleash its full potential.
\end{abstract}

Keywords: Vehicle-to-Everything Communication, connected cars, multistakeholder perspective, TOE framework.

\section{$1 \quad$ Introduction}

With an increasing number of sensors deployed in cars as well as a wide range of connectivity services offered, communication between vehicles, information systems, end devices, and humans operating in the road transportation system is increasing, named the Vehicle-to-Everything Communication (V2X) [1]. Studies show that by 2025 every new car will be connected to the Internet [2]. V2X can not only improve safety, increase traffic efficiency, but also be the foundation for diverse innovative services that will disrupt our understanding of road transportation [3]. Concurrently, increasing V2X implicates many new data streams exposing the automotive traffic and infrastructure to new risks and challenges [4]. For example, hackers were able to gain control over a Jeep Cherokee to track its travel route and remotely disable the breaks [5]. The faultless functionality of V2X depends on many different, cross-industry stakeholders resulting in a complex network of interdependencies and confronting companies with the difficulty of observing challenges pending that need to be solved.

$15^{\text {th }}$ International Conference on Wirtschaftsinformatik, March 08-11, 2020, Potsdam, Germany 
Different aspects of V2X are already addressed in prior research, for example, how to overcome certain challenges [6] and valuable use-cases of V2X [7]. Mainly technological challenges of V2X and potential solutions are examined, e.g. the challenge of data collection using a uniform mechanism and many heterogeneous sensors or the seamless integration of heterogeneous software elements [6]. Still, prior research states most challenges implicitly by proposing possible solutions but does not provide empirical confirmation. Likewise, we lack a deep understanding of their origins and consequences. Further research needs to assess V2X related challenges from a more holistic perspective and complement current findings by uncovering the interdisciplinary challenges of V2X. Therefore, this paper aims to answer the research question: What challenges of $V 2 X$ exist?

To identify V2X related challenges, we conducted comprehensive interviews with 19 industry experts from different industry sectors, including manufacturers and suppliers from the automotive sector, consultancies, and stakeholders from the telecommunication sector. We determined various challenges that we clustered into five major challenges. By taking the perspective of the Technology- OrganizationEnvironment (TOE) Framework [8], we also reveal that these challenges not only relate to technological issues but also problems within the organizations and environment. Identified challenges include economic risk, data security and privacy, and legal regulation, among others. With our study, we provide a more holistic overview of what challenges the implementation and diffusion of V2X are facing. With that, we guide future research towards emphasizing the relevant challenges and make practitioners more aware of what $\mathrm{V} 2 \mathrm{X}$ related challenges need to be tackled across industries.

\section{Theoretical Background}

\section{$2.1 \quad$ Vehicle-to-Everything}

The communication within a road transportation system is accumulated as V2X. This term sums up the entire communication spectrum, wireless communication via networks but also visual signals as flashing for example. V2X is commonly classified according to the link type, including Vehicle-to-Vehicle (V2V), Vehicle-toInfrastructure (V2I) and Vehicle-to-Pedestrian Communication (V2P) [3].

$\mathrm{V} 2 \mathrm{X}$ is divided into the three main components within the system, which are the vehicle, the communication technology, and the communication partner on the other end of the channel. As a communication partner, the different options such as pedestrian, vehicle, cloud, etc. are considered aggregated and technically not further specified within this paper. Although it is named V2X, the communication is considered bidirectional for this research and could be seen as Everything-to-Vehicle communication as well. To match the scope of this paper we focus on cars as particular vehicles and the WLAN-based and Cellular-based digital communication channels in the extended context of advanced driver assistance and autonomous systems.

The system architecture of an intelligent, connected car builds the base for the communication network. Within a cooperative car, sensor units are distributed all over the car to gather information from the environment [10]. Every sensor is connected to 
one or several Electronic Control Unit (ECU) via a bus system. These ECUs are computing devices where programs such as collision avoidance, cooperative driving and more, run on top of the stack. These programs process the data inputs from the numerous sensors. Based on the calculated results, the mechanical parts of the car are steered and the ECU transmits the data to the vehicle Application Control Unit (ACU) which each car holds. The ACU distributes the information to the car's environment.

The information which is sent and received by the ACU can be communicated through two main communication technologies, WLAN-based or Cellular-based. The WLAN-based technologies are operating in the $5.9 \mathrm{GHz}$ frequency band, also known as the $\mathrm{p}$ amendment of the IEEE 802.11p standard. It is also known as Dedicated ShortRange Communication (DSRC). The two most important standards are the Wireless Access in Vehicular Environment (WAVE) in the U.S. and the Cooperative Intelligent Transport System (C-ITS) in Europe [11]. Each car sends its information several times per minute and all surrounding communication partners, which are close enough, can receive the message. Nonetheless, there are only a few serial-produced cars with V2X radio chips, including Toyota Prius from 2015, GM Cadillac CTS from 2017, VW Golf from 2019. In the cellular-based technologies, the 3rd Generation Partnership Project published a standardization as Cellular Vehicle-to-Everything (C-V2X). The main advantage of $\mathrm{C}-\mathrm{V} 2 \mathrm{X}$ is the existing cellular infrastructure and the future evolution to $5 \mathrm{G}$, which envisions much higher bandwidth, as well as higher security and reliability [12]. Predictions are divided which technology will be predominant in the future since there is no clear commitment of the manufacturers and no universal standard yet.

\subsection{Related Work on Vehicle-to-Everything Challenges}

The potentials of V2X and suitable applications are growing rapidly [7] and with it its challenges. An excellent digest on state-of-the-art research as well as a brief historical context is provided by MacHardy et al. [13]. For this paper, we focus on existing literature regarding challenges related to V2X and connected cars as shown in Table 1.

Prior research has mostly taken a solely technical perspective on V2X and especially on its challenges (e.g. [7], [6]). For example, [7] compiles the current status in research in VANET technologies and is therefrom identifying open challenges for further research, including message broadcasting in vehicular networks. Research on technology challenges has focused on challenges with communication technologies (i.e., availability and latency) $[13,14]$ and underlying architecture and infrastructure (i.e., lack of source for synchronization) [15]. Prior research has also looked at engineering challenges that occur integrating connected vehicles into the IoT ecosystem, such as integrating all heterogeneous elements into a standard IoT architecture [6]. A report on the current status of DSRC development in the US gives a broad overview of different challenges not only from a technical and research-driven perspective. Nonetheless, the paper is focused on DSRC exclusively and geographically restricted to the US [16].

Summarizing the reviewed literature, there are many valuable surveys and reviews on existing V2X technology and emerging technical challenges. Nevertheless, the perspective is often restricted either to an exclusively technical focus or a focal point 
on the theoretical research aspects rather than the entire ecosystem respecting the different stakeholders. This paper closes the gap by analyzing the statements of different stakeholders from industry and research. The outcome provides challenges not only from a technical standpoint but also from an organizational and an environmental one.

Table 1. Overview of related research on V2X challenges.

\begin{tabular}{|c|c|c|c|c|c|}
\hline \multirow{2}{*}{ Ref. } & \multirow{2}{*}{$\begin{array}{l}\text { Communication } \\
\text { Technology }\end{array}$} & \multicolumn{3}{|c|}{ Focus } & \multirow{2}{*}{ Findings } \\
\hline & & $\mathbf{T}$ & $\mathbf{O}$ & $\mathbf{E}$ & \\
\hline$[7]$ & DSRC; Cellular & $\mathrm{X}$ & & & $\begin{array}{l}\text { Listing ten technical-focused research challenges in the context of } \\
\text { VANET derived from the current state of development. }\end{array}$ \\
\hline [13] & DSRC; Cellular & $\mathrm{X}$ & & $\mathrm{X}$ & $\begin{array}{l}\text { Survey technical developments and current research and deriving } \\
\text { challenges focused on communication technologies. }\end{array}$ \\
\hline$[6]$ & Not specified & $\mathrm{X}$ & & & $\begin{array}{l}\text { Formulation of technical research and engineering challenges for } \\
\text { the integration of connected vehicles in the IoT ecosystem. }\end{array}$ \\
\hline [14] & DSRC; Cellular & $\mathrm{X}$ & & $\mathrm{X}$ & $\begin{array}{l}\text { Discuss existing as well as developing V2X technologies and } \\
\text { identification of related major challenges. }\end{array}$ \\
\hline$[15]$ & DSRC; Cellular & $\mathrm{X}$ & & & $\begin{array}{l}\text { Focus on vehicular communication architectures and technologies } \\
\text { as well as their associated technical challenges. }\end{array}$ \\
\hline$[16]$ & DSRC & $\mathrm{X}$ & $\mathrm{X}$ & $\mathrm{X}$ & $\begin{array}{l}\text { Identification and valuation of several challenges concerning V2X } \\
\text { exclusively in the context of DSRC. }\end{array}$ \\
\hline \multicolumn{6}{|c|}{$\mathrm{T}=$ technical, $\mathrm{O}=$ organizational, $\mathrm{E}=$ environmental challenges } \\
\hline
\end{tabular}

\section{$3 \quad$ Research Method}

\subsection{Data Collection}

We applied an explorative and inductive research approach to validate and deepen our understanding of challenges derived from literature and to investigate complex circumstances that the literature has not yet explored [17]. Besides, research has shown explorative and inductive approaches to be useful when addressing new or poorly understood phenomena [18] and to consider the contexts that embed those phenomena [19].

We conducted 19 one-to-one interviews with experts in the V2X industry, to collect data for a better understanding of the problem domain and derive relevant challenges. Due to the inductive approach, we did not select theories to test or incorporate before collecting and analyzing data [20]. To identify different stakeholder groups a purposeful sampling was used, which is a method for the identification and selection of individuals with special expertise on a certain topic [21]. Four main stakeholder groups were identified to achieve holistic coverage of the different industries working on various aspects of V2X. Therefore, we have looked for people who either have many years of experience in the car or the communication industry or have major touchpoints with the V2X technology based on their current employment. The first two stakeholder 
groups stem from analyzing the three large associations in V2X technology - the 5GAA, the Linux Foundation, and the IEEE - which confirmed the relevance of the automotive industry as well as the infrastructure and telecommunication industry. The automotive industry is represented by interviews with three German car manufacturers, as well as four different suppliers, two of which provide simulation software and information systems for cars. The infrastructure cluster is represented by two interviews with experts from telecommunication companies. The two remaining clusters comprise additional stakeholders in one group, like research institutions, consultancies, and insurance companies, represented by interviews with five consultants working with V2X daily, as well as two researchers from a leading German technical university. In the last stakeholder group, we interviewed one start-up and two practitioners from Silicon Valley with a more independent and innovative perspective. Two of the experts also talked about their work for the 5GAA. This highly diverse group of practitioners helped to understand the problem domain and challenges regarding V2X. Table 2 outlines the distribution and demographics of the interviewees.

Table 2. Overview of demographics of the interview partners.

\begin{tabular}{cl}
\hline Characteristic & \multicolumn{1}{c}{ V2X Interviewees (n=19) } \\
\hline Gender & $89 \%$ male; 11\% female \\
Geographic location & $83 \%$ Germany; 12\% USA; 5\% UK \\
Educational qualification & $79 \%$ university degree; 16\% PhD; 5\% other \\
Years of job experience & $5 \%<1$ year; 37\% 1-5 years; 21\% 5-10 years; 37\% >10 years \\
$\begin{array}{c}\text { Organization size } \\
\text { (Number of employees) }\end{array}$ & $16 \%<10 ; 16 \% 10-500 ; 11 \% 501-5000 ; 26 \% 5001-100000 ; 31 \%>100000$ \\
Enterprise industry & $\begin{array}{l}37 \% \text { automotive industry, 37\% additional stakeholders, } 15 \% \text { innovation } \\
\text { sector, 11\% infrastructure }\end{array}$ \\
\hline
\end{tabular}

We applied a semi-structured interview method for three different reasons. Because this paper aims to find out challenges related to V2X as well as the industry representatives' awareness for them, a certain basic structure is necessary. Additionally, a semistructured interview makes the results more comparable. However, as the topic is cutting-edge, the semi-structured approach gives the experts the freedom to talk about things that might not have or could have been considered in the preparation of the interviews [22]. Thus, we conducted each interview based on an interview guide [23]. We asked interview partners about potential V2X challenges in the context of their work which occur in the connected infrastructure. After an introduction to the topic, we first asked open questions to avoid imposing our point of view and then, we asked questions following our semi-structured guideline referring challenges that were divided into technical, organizational and environmental factors. The interview guideline was derived and discussed by three researchers beforehand. Afterward, two senior editors were pre-testing the interview guideline whereas they assessed the comprehensibility and the structure of the guide. In addition, we made constant improvements to the questionnaire in terms of clarity and comprehensibility of the questions. Furthermore, we applied a non-judgmental form of listening, maintained distance, and strived to sustain an open and non-directive style of conversation during 
the interviews to ensure impartiality and avoid bias [22, 23]. We recorded and transcribed each interview except for five, for which we did not get the permission to record. For them, we took detailed field notes for further analysis. The expert's interviews lasted 35 minutes on average.

\subsection{Data Analysis}

For the transcribed interviews and field notes a three-stage coding approach was implemented: (1) open coding, (2) axial coding, (3) selective coding [24]. Coding refers to a process in which one annotates and labels interview transcripts with a piece of text [25]. To determine the labels, we used words that the interviewees suggested [26]. We used Atlas.ti 8, a coding tool for qualitative data. Starting with open coding entails fracturing the data by describing concepts in it that may define a significant occurrence or incident about a phenomenon [20,24]. During open coding, all available data were labeled for direct visibility of the structure and information of the interview. Additionally, the participants were classified into the various stakeholder groups. With open coding, 283 codes related to 451 textual segments were obtained from the 19 interviews (including field notes). For example, the statement of an interview "[...] the question of data privacy. All of these are unresolved issues" was coded as „Data Privacy".

In the second stage, axial coding was used to identify conditions, actions/interactions, and consequences [24]. Axial coding allows the comparison of codes to create hierarchical classifications by clustering codes according to common themes. In our case, axial coding was used to analyze the text segments in terms of conditions and consequences of challenges (as actions/interactions). Understanding conditions and consequences helped us to group similar codes to more abstract categories, such as the codes "Investment Costs for Infrastructure" and "Economic Efficiency" were grouped to "Economic Risk". During axial coding, initially, 24 groups of categories were identified, which were condensed into five major challenges.

Finally, we strived to move beyond a mere description of challenges to a more abstract level of conceptualization [27]. We applied selective coding to cluster the groups of categories even further, again creating hierarchical classifications [20, 24]. In particular, we reflected on the challenges by building on the theoretical foundations of the Technology-Organization-Environment (TOE) Framework [8], which describes aspects of an organization's context influencing the adoption of innovations. The three contexts are (1) organizational: characteristics and resources of an organization that have an impact on innovation adoption (e.g., top management support); (2) environmental: factors like the regulatory environment, the structure of the industry and the presence of technology providers; (3) technological context: including all technological aspects that are relevant to the organization (e.g., technology complexity). By applying selective coding, we mapped identified challenges to these dimensions, supporting the differentiation of challenges and highlighting organizational and environmental challenges that have been neglected by prior research. For example, acceptance was mapped to the dimension organization. In the following, we will discuss the most important challenges for each TOE dimension, according to the ranking of our interviewees. 


\section{Challenges of Vehicle-to-Everything Communication}

Based on the diverse stakeholder groups, we categorized the interview partners into six categories: car manufacturers [M] and suppliers [S] for the automotive industry, telecommunication providers [T] for infrastructure, innovative companies and startups [I] for the innovation sector and finally research [R] and consultancies [C].

\subsection{Technological Challenges}

Data security and privacy become an important topic with information being exchanged between vehicles and other stakeholders in the mobility infrastructure. To communicate with other stakeholders, vehicles have to establish a direct connection via a communication network. Because this connection is vulnerable to cyber-attacks, it poses a threat to the vehicles as well as its driver's data and cybersecurity [S; I; R]. Security can be compromised either by direct hacking attacks or just by an overload of the system [I; R]. "[Security] is a major challenge. There is a process chain of car, traffic infrastructure, cellular providers, short-distance communication, a lot of backends, a lot of data constellations like map updates, etc. [...] Of course there are many attack vectors." [T]. However, because vulnerabilities in the system will always exist, comprehensive security will never be given [S; C; M; T]. Nevertheless, security software needs to be developed to make attacks more difficult [I]. Experts from [T] observe that the government is interested in developing approaches for better protection as well. Yet, government involvement may slow down innovation processes [I].

With an increasing amount of data being collected data privacy gains in importance. In the context of V2X data privacy is an especially sensitive topic because mobility data specific to the individual reveals extremely sensible information as, for example, one's movement patterns. This might also open the possibility of data trading [S; M]. "And if it is a central infrastructure, then the guy really knows my complete motion profile. Do I want that?" [M]. Additionally, mobility and vehicle data of all kinds will most likely be stored in one single data lake and thus store a lot of information about individuals in one place $[\mathrm{S} ; \mathrm{C}$ ]. In the EU, the General Data Protection Regulation (GDPR), unambiguously and internationally regulates data protection of personal data. Yet, because the regulation leaves room for interpretation given technical neutrality in their requirements, and thus results in uncertainty, adhering to the GDPR is challenging for V2X infrastructure [M; S; C]. Overly strict regulations could also lead to data sparseness, complicating manufacturer's work [M]. Thus, additional research should be carried out to develop a more practical way of ensuring data privacy and preventing unrestrained mass surveillance. [T; M; C].

\subsection{Environmental Challenges}

Economic risk is another challenge identified by the interviewees, more precise the difficulty of controlling investment in infrastructures. Transportation in combination with a standardized telecommunication infrastructure is a prerequisite for $\mathrm{V} 2 \mathrm{X}$ technology. While cost reduction potential is seen in, for example, making traffic lights and similar devices dispensable with V2X, interviewees also stated that the 
transportation infrastructure is already in an investment backlog [I; C]. Hesitant investment decisions determined by slow decision-making processes are hindering the advancement of new technologies [I]. However, since not only the telecommunications companies are interested in maintaining their competitive position, but also car manufacturers and local authorities, the question arises as to who will pay for the expansion [C; M; T]. "I see three big topics [...] and the third topic is: who will pay for it?" [T]. Many interview partners regard it as the task of municipalities or other government institutions to provide a compatible infrastructure. Simultaneously, it is perceived as difficult for a single party to bear the investment costs by itself [T; M].

Internationalization is of big importance as a vehicle is a means of transport enabling individual mobility in- and outside of country borders. Therefore, the seamless international application of V2X is crucial. Because not only transmission technologies and legislation but also communication protocols differ greatly between countries, regions and even cities, agreeing on a common international standard technologically as well as legally poses a challenge for $\mathrm{V} 2 \mathrm{X}[\mathrm{S} ; \mathrm{T} ; \mathrm{C} ; \mathrm{M}]$. "Even if you have the same protocol, it can be interpreted differently, depending on how well it is specified. This is one of the biggest challenges related to [V2X]. Depending on which country one is driving, or even which city, a very diverse range of topics emerges. One city behaves completely different from another." [M]. The absence of international standards may lead to a delay in the transition from one standard to another while crossing borders which can cause downtime and failures [T]. So far, international cooperation on the subject is rare. However, the EU is perceived as an opportunity to develop a standard framework for connected driving and transport in an international context of different countries [I]. While the establishment of worldwide standards is considered best, a split into sub-markets such as America, Europe, and China is also seen as a solution [C; S].

Legal Regulation and Responsibility reoccur in many other topics and possess one of the biggest challenges for V2X currently, as perceived by the interviewees. Different aspects of V2X demand clear legislation and regulation. Telecommunication companies are waiting for government institutions to give a clear statement about whether the infrastructure is going to be privatized or public. Car manufacturers and suppliers, on the other hand, are waiting to see whether a comprehensive infrastructure will be implemented and if so, which communication technology it will support. The opinions are divided on whether the infrastructure should be operated privatized or publicly [R; S; C]. While complete public operation might foster inefficiencies and slow down the innovation process, many interviewees agree that public institutions should at least have a say in the administration of the infrastructure [M; T; S]. Besides the infrastructure topic, the legal liability of the different stakeholders for different usecases of V2X was addressed by the interviewees. One prominent example is autonomous driving. Both, telecommunication companies as well as car manufacturers and suppliers demand clear regulations on who will be held responsible in case of an accident due to a communication failure [T; M]. While some say, manufacturers should be held responsible since they sell the product, others say telecommunication companies will be responsible for ensuring a resilient network [S; R]. A third option could be to let independent auditing firms assess the security and with that take part of the responsibility $[\mathrm{S}]$. However, interviewees regard politics as responsible to answer 
the ethical question linked to this topic. "If you take the Kobayashi-Nero-Scenario, then one will have to answer the ethical question of whether to drive into a group of students or a group of retirees. Or to do the Harakiri and drive into a tree." [T]. If V2X ends up being part of every car but provides redundancy for sensor data instead of serving as the main base for critical decisions, this challenge might become negligible. [M; R].

\subsection{Organizational Challenges}

Acceptance, which is very low, and trust among potential users as well as manufacturers is another challenge of V2X. Because many issues remain regarding legislation, international communication standards, and communication technology, all stakeholders are hesitant to invest in V2X without clearly knowing different use cases and how they can be monetized [M]. At the same time, users do not trust the technology yet and thus have a low willingness to pay for its implementation. "The general public is not yet very aware of the topic (V2X). [...] I think some time is going to pass before it really explodes." [M]. Also, users perceive other vehicle-related features as more important, as e.g. low emissions. This results in a deadlock since V2X based services will work best with a broad acceptance among users, which can only be won through investing in the development of value-adding services [I]. A way to counteract this issue could be higher media attention [M]. Additionally, the trust will grow if users feel they can rely on their providers, e.g. in terms of trustworthy data usage [T].

\section{Discussion}

\subsection{Discussion of Findings}

$\mathrm{V} 2 \mathrm{X}$ is a relevant and widely discussed topic within literature and, as our interviews showed, within the industry as well. Despite this, V2X is rarely discussed concerning the surrounding infrastructure, especially regarding infrastructure which might need to be created within this evolving and, so far, steadily changing ecosystem. We used interviews with industry experts to systematically collect challenges of V2X and the related infrastructure, which are, if any, mentioned implicitly and partially in the existing literature. The main reason for the lack of structural elaboration on possible challenges is evident in both, literature and the conducted interviews. V2X must be seen as a technology pushed onto the market. The development is mainly driven by technology and thereof arising opportunities. Actual applications and value-adding use cases, which generate significant market interest are developed incrementally within current technological possibilities. In contrast, for a technology pulled by the market, challenges that are solved by the technology and therefore also the challenges which need to be solved during the development of it would be identified quicker. Further technological progress must identify useful and economically reasonable applications, as with other new technologies. Otherwise, the development is slowed down as literature and our interview partners agree on.

For the conducted interviews, we identified several further challenges, which were mentioned implicitly and explicitly by the experts, as well as interdependencies 
between challenges. For example, the challenge of a reliable technical foundation, which was also mentioned within most of our interviews, gets evident discussing the underlying technology. The interviewees have diverging opinions and are to some extent doubtful whether WLAN or cellular technology should be the one to move forward with. As of today, WLAN is the only technology providing reliable applications, but most of the experts agree that only cellular, which is still unreliable, can fulfill the demands of the future. Additionally, responsibility and legal regulations become important with regards to the security of each technology and the systems it is used in. Furthermore, they could influence internationalization in several ways. Existing national laws and regulations could provide a constructible structure for international guidelines. On the other hand, it could complicate the process of finding international standards if national guidelines will be established first and they differ too much between nations.

Within the interviewed stakeholders, we identified two contrary positions concerning the significance of V2X for the future. Innovative and young companies see $\mathrm{V} 2 \mathrm{X}$ technology as unnecessary in the future. Fully autonomous driving would mean that there would no longer be any communication between the vehicles based on an information infrastructure since the intelligence would lie in the backend inside the car. The intelligence will make it possible that only optical communication and perception of the cars is required, and no digital information communication has to be available. Through these autonomous features, many challenges, such as the reliability and responsibility of infrastructure, would become obsolete. On the other hand, car manufacturers, telecommunications companies and associations have underlined the importance of V2X technology. They present the V2X technology as an important, under certain circumstances also transitional technology for autonomous driving. The different approaches and different perceptions among the stakeholders emphasize challenges regarding acceptance because it shows that there are uncertainties to be solved and solutions to be found.

\subsection{Implications, Limitations of Study and Future Work}

We contribute to practice and research in several ways. Important challenges are highlighted and we call for future research, for example developing innovative security and privacy means. In particular, we extend the hitherto view from a technologyindependent perspective on technical challenges in prior research by adding environmental and organizational challenges as well. We thus want to raise red flags, that researchers and practitioners should not solely focus on technological issues, but rather focus on the demanded solutions and needs to further push the acceptance and therefore diffusion of the technology. To counteract current shortcomings a holistic approach of V2X communication as an integral part of the transportation infrastructure is required. Interviewing different stakeholders provides practice with a good overview of major challenges in the $\mathrm{V} 2 \mathrm{X}$ ecosystem. We contribute the required context to not only grasp existing interdependencies between several topics and the related challenges but also provide recommendations on how to tackle challenges.

Nevertheless, our study has some limitations. First, regarding the number and selection of interview partners. So far 19 interviews have been conducted. Although the 
aim was to cover as many different stakeholders as possible, some stakeholder groups are represented more strongly than others as well as a geographical overrepresentation of Germany. Consequently, the results give an initial overview, but generalization must be savored with caution. Second, the depth of the interviews limits the results. The purpose of the interviews was to collate various challenging aspects to cluster them into challenge groups conclusively. This is to the expense of the scientific depth of the interviews. The holistic analysis and solution finding of the mentioned aspects were not in the scope of the interviews. Besides, the resulting challenge groups were derived from the different interviews and only to some extent the perception of the aspects as challenges where explicitly discussed with the interviewees.

Our results pave the way for several future research opportunities. On the one hand, qualitative research methods could be used to further specify the identified challenges, cross-check their acceptance within the stakeholder groups and carve out appropriate solutions. Beyond that, the in-depth analysis of the challenges could provide the foundation for weighting the significance of them as well as identify further strains.

\section{Conclusion}

Conducting 19 semi-structured interviews with experts from multiple different market players revealed that $\mathrm{V} 2 \mathrm{X}$ is perceived as a critical information infrastructure coming with several challenges that must be tackled. These challenges are clustered as data security and privacy, economic risk, internationalization, legal regulation and responsibility, and acceptance. Considering predominant market mechanisms, the conflict between technological possibilities and economical appliance, which is reflected by the identified challenges, does not surprise. Coming back to the statement that by 2025 all cars will be connected to the internet, this point is further demonstrated. Internet is used not only for advanced driver assistance and autonomous systems but also for infotainment. In this context, some challenges are not as strong or already been solved, because of similarities to other fields of application and therefore transferable solutions. That leads to a broader acceptance, which offers the industry the economic attractivity to deploy the technology. In contrast, the application of the technology as discussed in this paper is not only a completely new field of application but also an evolving infrastructure considered critical. Therefore, transferable knowledge is hard to gather. This slows down the market penetration and makes the technology less attractive for industry to use. Through clearly stating major challenges, we provide recommendations and a point of departure for future research avenues to overcome existing challenges and enable widespread diffusion of $\mathrm{V} 2 \mathrm{X}$ to unleash its full potential.

\section{References}

1. Fallgren, M., Dillinger, M., Alonso-Zarate, J., Boban, M., Abbas, T., Manolakis, K., Mahmoodi, T., Svensson, T., Laya, A., Vilalta, R.: Fifth-Generation Technologies for the Connected Car. IEEE Veh. Technol. Mag. 13, 28-38 (2018)

2. McCarthy, N.: Connected Cars By The Numbers, https://www.forbes.com/sites/ niallmccarthy/2015/01/27/connected-cars-by-the-numbers-infographic/\#\#5c02b1 fe1028 
3. Boban, M., Kousaridas, A., Manolakis, K., Eichinger, J., Xu, W.: Connected Roads of the Future. IEEE Veh. Technol. Mag. 13, 110-123 (2018)

4. Rizvi, S., Willet, J., Perino, D., Marasco, S., Condo, C.: A Threat to Vehicular Cyber Security and the Urgency for Correction. Procedia Computer Science 114, 100-105 (2017)

5. Miller, C. and Valasek, C.: Remote Exploitation of an Unaltered Passenger Vehicle, http://illmatics.com/Remote\%20Car\%20Hacking.pdf

6. Datta, S.K., Da Costa, R.P.F., Harri, J., Bonnet, C.: Integrating connected vehicles in Internet of Things ecosystems. In: Proc. IEEE 17th International Symposium on WoWMoM, pp. 1-6 (2016)

7. Eze, E.C., Zhang, S.-J., Liu, E.-J., Eze, J.C.: Advances in vehicular ad-hoc networks (VANETs). Int. J. Autom. Comput. 13, 1-18 (2016)

8. Tornatzky, L.G., Fleischer, M.: The processes of technological innovation. Lexington Books, Lexington, Mass. (1990)

9. Corchero, C., Sanmarti, M.: Vehicle- to- Everything (V2X). In: Proc. 15th International Conference on the European Energy Market, pp. 1-4 (2018)

10. Alesiani, F., Gajek, S.: Remote Testimony. In: Proc. IEEE 83rd Vehicular Technology Conference, pp. 1-5 (2016)

11. Festag, A.: Standards for vehicular communication-from IEEE $802.11 \mathrm{p}$ to $5 \mathrm{G}$. Elektrotech. Inftech. 132, 409-416 (2015)

12. Abboud, K., Omar, H.A., Zhuang, W.: Interworking of DSRC and Cellular Network Technologies for V2X Communications. IEEE Trans. Veh. Technol. 65, 9457-9470 (2016)

13. MacHardy, Z., Khan, A., Obana, K., Iwashina, S.: V2X Access Technologies. IEEE Commun. Surv. Tutorials 20, 1858-1877 (2018)

14. Bey, T., Tewolde, G., Kwon, J.: Short Survey of Vehicular Communication Technology. In: Proc. IEEE International Conference on EIT, pp. 515-518 (2018)

15. Singh, P.K., Nandi, S.K., Nandi, S.: A tutorial survey on vehicular communication state of the art, and future research directions. Vehicular Communications 18, 100164 (2019)

16. Review of the USDOT Report on Connected Vehicle Initiative Communications Systems Deployment. Transportation Research Board, Washington, D.C. (2015)

17. Kuechler, W.L., Park, E.H., Vaishnavi, V.K.: Formalizing Theory Development in IS Design Science Research. In: Proc. AMCIS (2009)

18. Smolander, K., Rossi, M., Purao, S.: Software architectures. Blueprint, Literature, Language or Decision? European Journal of Information Systems 17, 575-588 (2008)

19. Volkoff, O., Strong, D.M., Elmes, M.B.: Understanding enterprise systems-enabled integration. European Journal of Information Systems 14, 110-120 (2005)

20. Abraham, C., Boudreau, M.-C., Junglas, I., Watson, R.: Enriching our theoretical repertoire. European Journal of Information Systems 22, 56-75 (2013)

21. Palinkas, L.A., Horwitz, S.M., Green, C.A., Wisdom, J.P., Duan, N., Hoagwood, K.: Purposeful Sampling for Qualitative Data Collection and Analysis in Mixed Method Implementation Research. Administration and policy in mental health 42, 533-544 (2015)

22. Myers, M.D.: Qualitative research in business \& management. Sage Publ, London (2013)

23. Yin, R.K.: Case study research. Design and methods. SAGE, Los Angeles, London, New Delhi, Singapore, Washington, DC (2014)

24. Corbin, J.M., Strauss, A.L.: Basics of qualitative research. SAGE, Los Angeles, London, New Delhi, Singapore, Washington DC, Boston (2015)

25. Jones, S., Hughes, J.: Understanding IS evaluation as a complex social process. European Journal of Information Systems 10, 189-203 (2001)

26. Volkoff, O., Strong, D.M., Elmes, M.B.: Technological Embeddedness and Organizational Change. Organization Science 18, 832-848 (2007)

27. Urquhart, C., Lehmann, H., Myers, M.D.: Putting the 'theory' back into grounded theory. Information Systems Journal 20, 357-381 (2010) 